\title{
A Study of Pressure Gradient in Multiphase Flow in Vertical Pipes
}

\author{
David Nwobisi Wordu, Felix J. K. Ideriah, and Barinyima Nkoi
}

\begin{abstract}
The study of multiphase flow in vertical pipes is aimed at effective and accurate design of tubing, surface facilities and well performance optimization for the production of oil and gas in the petroleum industry by developing a better approach for predicting pressure gradient. In this study, field data was analyzed using mathematical model, multiphase flow correlations, statistical model, and computer programming to predict accurately the flow regime, liquid holdup and pressure drop gradient which are important in the optimization of well. A Computer programme was used to prediction pressure drop gradient. Four dimensionless parameters liquid velocity number (Nlv), gas velocity number (Ngv), pipe diameter number (Nd), liquid viscosity number (NI), were chosen because they represent an integration of the two dominant components that influence pressure drop in pipes. These dominant component are flow channel/media and the flowing fluid. The model was found to give a fit of $100 \%$ to the selected data points. Hagedorn \& Brown, Griffith \&Wallis correlations and model were compared with field data and the overall pressure gradient for a total depth of $10000 \mathrm{ft}$ was predicted. The predicted pressure gradient measured was found to be $0.320778 \mathrm{psi} / \mathrm{ft}$, Graffith\& Wallis gave 0.382649Psi/ft, Hagedorn \& Brown gave 0.382649Psi/ft; whereas generated model gave $0.271514 \mathrm{Psi} / \mathrm{ft}$. These results indicate that the model equation generated is better and leads to a reasonably accurate prediction of pressure drop gradient according to measured pressure gradient.
\end{abstract}

Index Terms-Two-Phase Flow; Pressure Drop Gradient; Flow Regimes; Gas-Liquid Ratio; Dimensionless Groups; Regression Analysis.

\section{INTRODUCTION}

Multiphase flow is a simultaneous flow of materials with different phases or states flowing together. The heterogeneous occurrence of two phase and three phase flows in pipes is much more complex than single-phase flow. The phases tend to separate because of difference in density. Shear stresses at the pipe are different for each phase as a result of different densities and viscosities. The volumetric gas flow rate will be increased by decreasing pressure along the pipe and increasing the volume occupied by the compressible gas phase [1]. The gas and liquid phases do not travel at the same velocity in the pipe, the less dense, more compressible, less viscous gas phase tend to flow at high velocity than the liquid phase thereby causing slippage.

Reference [2] worked on optimization of flow line design for a two- phase flow of oil and gas in Nigerian oil fields. The study determined the optimal equation for the pressure drop along horizontal flowlines from oil wells to the flow

Published on January 23, 2019.

Authors are with the Department of Mechanical Engineering, Rivers State University, Nigeria. (e-mail: davidwordu85@gmail.com) station. This is of national economic importance for pipe sizing, pipe selection and optimization of flowline.

How to accurately estimate and minimizing Pressure Losses to Improve Well Productivity and optimization is key in study of multiphase flow in pipes. When oil is produced through the tubing, it experiences pressure drop. This pressure loss increases as the oil and gas moves higher in the tubing, this reduction in flowing pressure will cause more dissolved gas to come out of solution. Consequently, the flow stream, especially free gas, expands in volume per unit flow rate. The loss in pressure between the reservoir and the surface facilities leads to the reduction in the volume of oil that can be produced from the initial oil in place. Pressure loss in the production system has been a huge challenge in the transportation of reservoir fluids to the surface. This has attracted different investigative approach to study how to reduce pressure loss in the production system, which is vital to the volume of reservoir fluids produced at the surface [3].

Reference [4] did a comprehensive study on current pressure drop calculation on multiphase flow in vertical wells, current trends and future perspective. Based on the analysis the artificial neutral network models had showed better prediction accuracy and minimum number of variable even if other data beyond the range is used.

Reference [5] worked on detailed analysis of pressure drop in large diameter pipe; he found out that the measured total pressure drop and the calculated total pressure drop follow the same trend. But all the six empirical correlations selected slightly over-predicted the total pressure drop.

Predicting pressure losses in multiphase flow systems is not recent to the industry and it still does not have a complete solution that can cater for all types of flow conditions. This has brought about many particular solutions for limited flow conditions. This is as a result of the intricacy of multiphase flow and also the difficulty in analyzing even flow conditions that are limited. The model estimates the Pressure losses in conduit as the fluid flows through the pipe [6].

In order to accurately design and operate oil production facilities in an optimized means, it is necessary to predict the behavior of the two-phase flow of hydrocarbon in pipes with different angles. Practically all oil well production design involves evaluation of flow lines under two-phase flow conditions. However, the uncertainties in flow regime determination greatly affect the pressure drop predictions [7].

In multiphase flow pressure drop calculations, the total pressure gradient is divided into three com friction, elevation, and acceleration components. Each is calculated separately and then summed. For accurate calculation of the 
pressure gradient, particularly in long pipes, the length is divided into many small increments. An iterative calculation for each pipe segment is performed starting from the end of the pipe with the known pressure and progressing downstream. The total pressure gradient of each segment is multiplied by its length to yield the segment's pressure drop. The sum of the total segment pressure drops yields the pipeline's pressure drop. Because of complexities of twophase flow for performance of such calculations, it is necessary to use semi empirical or empirical correlations. These correlations fall into three classes: Homogeneous models, assuming that the gas and liquid phases travel at the same velocity (there will be no slippage). Consideration is not given to the flow regime. Separated models, assuming that the gas and liquid phases have different velocities (i.e., slip is taken into account). Attention is not given to flow pattern. Flow regime models, applying the same principles as separated models. However, the flow pattern is taken into account [8].

\section{MATERIAL AND METHODS}

\section{A. Materials}

The research methodology involves data collection and analysis. The field data is gotten from a vertical oil well for this work, other relevant parameters that cannot be measured directly were calculated using flow equations/ appropriate hydrodynamic equations.

\section{B. Methods}

The analysis of two-phase gas-liquid flow in pipes were carried out employing the physics of flow and flow equations

\section{1) Flow equations}

The basic equations governing fluid flow are

conservation of mass (continuity equation), conservation of momentum (Navier Stokes equations) and conservation of energy.

\section{2) Conservation of mass}

For control volume the mass in, minus the mass out, must equal the mass accumulation, according to [2].

$$
\frac{\partial p}{\partial t}+\frac{\partial}{\partial L}(\rho v)=0
$$

For steady state flow, no mass accumulation can occur.

$$
\frac{d}{d L}(\rho v)=0
$$

\section{3) Conservation of Momentum}

Rate of momentum out, minus the rate of momentum in, plus the rate of momentum accumulation in a given pipe segment must equal the sum of all the forces on the fluids. According to [9]: $\frac{\partial}{\partial t}(\rho v)+\frac{\partial}{\partial L}\left(\rho v^{2}\right)=-\frac{\partial p}{\partial L}-\tau \frac{\pi d}{A}-\rho g \sin \theta$

Steady state flow, mechanical energy balance equation becomes

$\frac{d p}{d L}=-\tau \frac{\pi d}{A}-\rho g \sin \theta-\rho v \frac{d v}{d L}$

Pressure gradient

$\frac{d p}{d L}=\frac{f \rho v 2}{2 d}+\rho g \sin \theta+\rho v \frac{d v}{d L}$

Neglecting acceleration terms

a. pressure gradient equation for single flow

$\frac{d p}{d L}=\frac{g}{g_{c}} \rho \sin \theta+\frac{f \rho v^{2}}{2 g_{c} d}$

Acceleration component of pressure gradient equation, the convective acceleration is usually neglected.

b. Reynolds number

$$
N_{\mathrm{Re}}=\frac{\rho v d}{\mu}
$$

\section{c. Turbulent Flow}

Friction factor can be gotten from equation or from moody chart

$$
\frac{1}{\sqrt{f}}=1.74-2 \log \left(\frac{\varepsilon}{d}+\frac{21.25}{N_{\mathrm{Re}}^{0.9}}\right)
$$

\section{4) Two Phase}

Multiphase flow in pipes, the pressure gradient equation for single phase is modified, to account for two phase flow in pipes.

a. Liquid holdup

Gas holdup or gas void fraction is expressed as

$H_{g}=1-H_{l}$

No-slip liquid holding (gas and liquid travelled at the same velocity)

b. Two-phase density

$$
\rho_{s}=\rho_{1} H_{L}+\rho_{g} H_{g}
$$

c. Multiphase Velocity

Two phase velocity

$$
V_{m}=V_{s L}+V_{s g}
$$




\section{5) Multiphase Viscosity}

The viscosity of the flowing fluid is used in determining a Reynolds number as well as other dimensionless number. The viscosity of an oil water mixture is the water oil ratio as a weighting factor

Two - phase viscosity

$$
\mu_{n}=\mu_{l} \lambda_{l}+\mu_{g} \lambda_{g}
$$

\section{Interfacial tension}

The interfacial tension between water and natural gas at various pressure and temperature depends on oil gravity.

$$
\sigma_{L}=\sigma_{0} f_{0}+\sigma_{w} f_{w}
$$

Where

$$
\begin{array}{lll}
\sigma_{0} & = & \text { oil surface tension } \\
\sigma_{w} & = & \text { water surface tension } \\
f_{0} & = & \text { oil fraction } \\
f_{w} & = & \text { water fraction }
\end{array}
$$

Modification of the pressure gradient equation for twophase flow for any fluid flowing in a pipe inclined, assuming that the gas-liquid mixture is considered homogenous over a finite volume of the pipe, neglecting the acceleration term.

$$
\frac{d p}{d L}=\frac{g}{g_{c}} \rho_{m} \sin \theta+\frac{f_{t p} \rho_{m} v_{m}^{2}}{2 g_{c} d}
$$

$$
\begin{aligned}
& \text { Where } \\
& \frac{d p}{d L}=\quad \text { pressure drop, } \mathrm{psia} / \mathrm{ft} \\
& \rho_{m}=\quad \text { mixture density } 1 \mathrm{bm} / \mathrm{ft} 3 \\
& V_{s g}=\text { gas superficial velocity, } \mathrm{ft} / \mathrm{sec} \\
& d=\text { pipe diameter, } \mathrm{ft} \\
& f_{t p}=\quad \text { Two phase friction factor } \\
& g_{c}=\quad \text { Conversion constant (32.17) Ibmft/Ibf } \\
& g=\text { acceleration due to gravity } \mathrm{ft} / \mathrm{sec} 2
\end{aligned}
$$

\section{Empirical Methods}

The method uses the general mechanical energy balance and the average in-situ density to calculate the pressure gradient. Hagedorn \& Brown and Griffith \& Wallis correlations will be used to predict the pressure drop gradient in this work, they are mathematically expressed below.

\section{1) Hagedorn \& Brown}

Hagedorn \&Brown used average pressure over the pipe length in calculating pressure gradient .

$\frac{d p}{d L}=\frac{g}{g_{c}} \rho_{m}+\frac{f_{t p} \rho_{f} v_{m}^{2}}{2 g_{c} d}$
Dimensionless groups proposed by Duns and Ros, (1963) were used to generate model equation. These groups can be modified to include constants that make them dimensionally consistent when common oilfield units are used.

\section{2) Liquid velocity number}

$N_{l v}=V_{s L} \sqrt[4]{\frac{\rho_{L}}{g \sigma_{L}}}$

3) Gas velocity number

$$
N_{l v}=1.938 V_{s L} \sqrt[4]{\frac{\rho_{L}}{\sigma_{L}}}
$$

4) Pipe diameter number

$$
N_{d}=d \sqrt{\frac{\rho_{l} g}{\sigma_{l}}}
$$

\section{5) Liquid viscosity number}

$$
N_{L}=\mu_{l} \sqrt[4]{\frac{g}{\rho_{l} \sigma_{l}}}
$$

\section{6) Griffith \&Wallis}

The Griffith correlation uses different holdup correlation, bases the frictional pressure gradient on in-situ average liquid velocity.

$$
\frac{d p}{d L}=\frac{g}{g_{c}} \rho-\frac{2 f \rho_{l} v_{l}^{2}}{2 g_{c} d}
$$

For field units

$$
\frac{1 d p}{144 d L}=\frac{g}{g_{c}} \rho^{-}+\frac{f m_{l}^{2}}{7.413 * 10^{10} * d^{5} \rho_{l} * H_{l}}
$$

Where

$\rho_{l}=$ liquid density $1 \mathrm{bm} / \mathrm{ft} 3$

$\rho^{-}=$mixture density $1 \mathrm{bm} / \mathrm{ft} 3$

$m_{l}=$ liquid mass flow rate $1 \mathrm{bm} / \mathrm{ft}$

\section{Model Equation Generated from Regression}

Model equation generated is a better approach for predicting pressure gradient in multiphase flow in vertical well. Four dimensionless groups (liquid velocity number $\mathrm{Nlv}$, gas velocity number $\mathrm{Ngv}$, pipe diameter number $\mathrm{Nd}$ and liquid viscosity number $\mathrm{Nl}$ ) were chosen which represent integration of flow parameters and flow media (density, velocity, viscosity, gravity, surface tension and 
pipe diameter). Statistical analysis was done, several models were generated, quadratic model gave the best, quadratic regression

Accurate prediction of pressure drop in vertical multiphase flow is needed for effective design of tubing and optimum production strategies. The complexity of the pressure drop calculation of two-phase flow systems is due to the variations in the gas and liquid flow rates across the two-phase flow stream. Two phase flow occurs during the production of oil and gas in the wellbore. Modeling this phenomenon is important for monitoring well,

Regression analysis was done to model the relationship between pressure drop and four dimensionless groups which represent multiphase flow variables. The model generated is given as follows:

$$
\begin{aligned}
& \mathrm{P}_{\text {red }}=349666.817+29792.358 \mathrm{~N}_{\mathrm{lv}}-2.478 \mathrm{~N}_{\mathrm{gv}}- \\
& 26141.966 \mathrm{~N}_{\mathrm{d}}-74.877 \mathrm{~N}_{\mathrm{l}}-2960.453 \mathrm{~N}_{\mathrm{lv}}^{2}-2.93 \times \\
& 10^{-2} \mathrm{~N}_{\mathrm{gv}}^{2}+406.22 \mathrm{~N}_{\mathrm{d}}^{2}+1563.72 \mathrm{~N}_{\mathrm{l}}^{2}
\end{aligned}
$$

The model was found to give a fit of $100 \%$ to the selected data point. Dimensional analysis was performed for the pressure drop and four dimensionless groups chosen which represented all the fluid properties and pipes used in this study and they are dimensionally correct.

\section{RESULTS AND DISCUSSION}

\section{A. Multiphase Flow Calculation}

The data used for this study was obtained an oil company in Port Harcourt, Niger Delta region. Multiphase flow pressure drop gradient prediction, it is important to determine the pressure volume temperature (PVT) properties, flow regimes, flow parameters using correlations or from field, pressure gradient in pipes is divided into elevation, friction and acceleration components. Each is calculated separately and then summed. For accurate estimation of the pressure gradient, particularly in long pipes, the length is divided into many small increments. An iterative calculation for each pipe segment is performed starting from the end of the pipe or location with the known pressure to the other end.

TABLE I: WELL

\begin{tabular}{ll}
\multicolumn{2}{c}{ TABLE I: WELL } \\
\hline Parameters & Values \\
\hline Length of tubing, ft & 10000 \\
Tubing size, in & 2.5 \\
Surface tension, dyne/cm & 30 \\
Water gravity, g/cc & 1.074 \\
Solution GOR, scf/STB & 400 \\
Surface temperature, of & 60 \\
Bottom hole temperature, of & 220 \\
Well head pressure, psi & 114.7 \\
Bottom hole pressure, psi & 3322.44 \\
Pipe roughness, ft & 0.0006 \\
Gas flow rate, Scf/day & 19262.82 \\
Liquid flow rate, bbl/day & 1000 \\
WOR & 1 \\
Gas density, Ibm/ft^3 & 0.148 \\
Liquid density, Ibm/ft^3 & 54.72 \\
Gas viscosity, cp & 0.016 \\
Liquid viscosity, cp & 0.889 \\
Gas gravity, cp & 0.78 \\
Gas FVF, Cuft/scf & 0.00536 \\
Oil FVF, bbl/STB & I.285 \\
\hline \hline
\end{tabular}

\section{B. Generated Model}

A computer programme was written to compute the fluid properties. To prepare this information, a case study well is chosen. The details of the well are displayed above in Table I.

Based on the fluid properties estimated, the flow properties are computed. These include the flow rates and flow velocities profile, as well as the fluid densities. Following the flowing properties, the programme estimates the flow regime as defined by the Griffith \& Wallis and Hagedorn \& Brown correlations respectively. This is important because the formula used for the subsequent pressure gradient computation depends on the prevalent flow regime. From the Spreadsheet programme, the pressure at different depths were computed and compared to the pressure obtained from measurement.

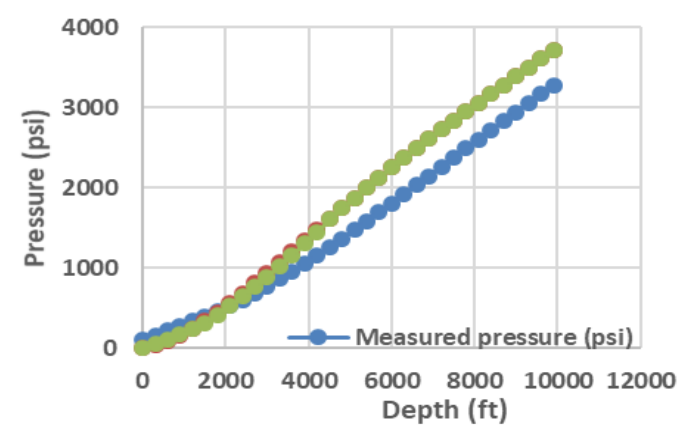

Fig. 1. Pressure profile in case study well.

From Fig. 1, it can be seen that both correlations had better agreement with the measured pressure closer to the surface (lower depth values). However, a big deviation is observed at deeper points in the well. This implies that the correlations tend to over - predict the pressure at the flowing pressure at deeper points in the well. As such, their use at such depths should be with caution.

\section{Regression Analysis}

A regression modelling approach was adopted for the modelling phase in this work. To successfully apply this, and verify the accuracy of the model generated, modelling approach was adopted. The modelling basically involves the use of selected data points from the population of study to build a model. To verify the accuracy of the model built, it is used to predict the variable values for the other points not used in the modelling. If inaccurate, the model is "trained" to fit the data points. This technique is used in the regression modelling adopted in this work. A regression model is fitted to some of the data points in Table I. The model is then used to predict the values of the used and unused data points. If accurate, it should be able to predict both sets of data points accurately. To achieve this, four regression models are used: multilinear, quadratic, cubic and exponential regression models. For all cases, the pressure in the pipe was correlated with four dimensionless variables.Liquid velocity number, Nlv, Gas velocity number, Ngv, Pip diameter number, Nd, Liquid viscosity number, N1. These dimensionless parameters were chosen because they represent an integration of the two dominant components that influence pressure drop in pipes: the flow channel/media and the flowing fluid. 


\section{1) Quadratic Regression}

For the quadratic regression, a similar approach is followed as in the multilinear regression. For this case, the following points were used to build the model. The model generated is given as follows:

$\mathrm{P}_{\mathrm{red}}=349666.817+29792.358 \mathrm{~N}_{\mathrm{lv}}-2.478 \mathrm{~N}_{\mathrm{gv}}-$ $26141.966 \mathrm{~N}_{\mathrm{d}}-74.877 \mathrm{~N}_{\mathrm{l}}-2960.453 \mathrm{~N}_{\mathrm{lv}}^{2}-2.93 \times$ $10^{-2} \mathrm{~N}_{\mathrm{gv}}^{2}+406.22 \mathrm{~N}_{\mathrm{d}}^{2}+1563.72 \mathrm{~N}_{\mathrm{l}}^{2}$

The model was found to give a fit of $100 \%$ to the selected data points and other goodness of fit parameters. It can be seen that there is considerably lower error in the quadratic model. Finally, to verify its applicability to other points that were used in building the model, it was applied to predict the pressure in the pipe, all points considered

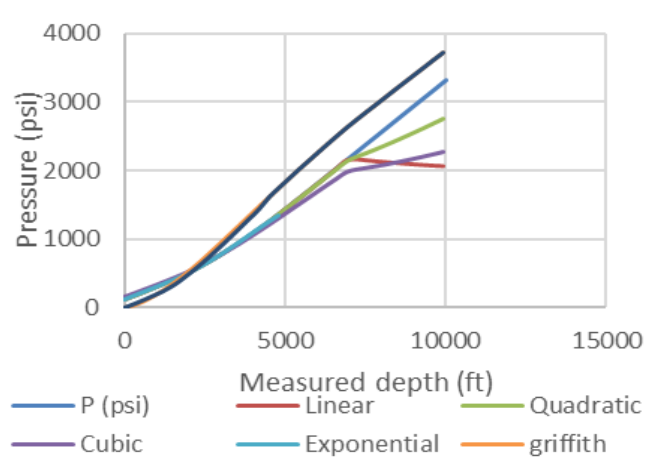

Fig. 2. Comparison of pressure drop using various regression models and multiphase flow correlations.

It can be seen that of all the models compared, the quadratic model was the most accurate. It gave a closer match to measured pressure.

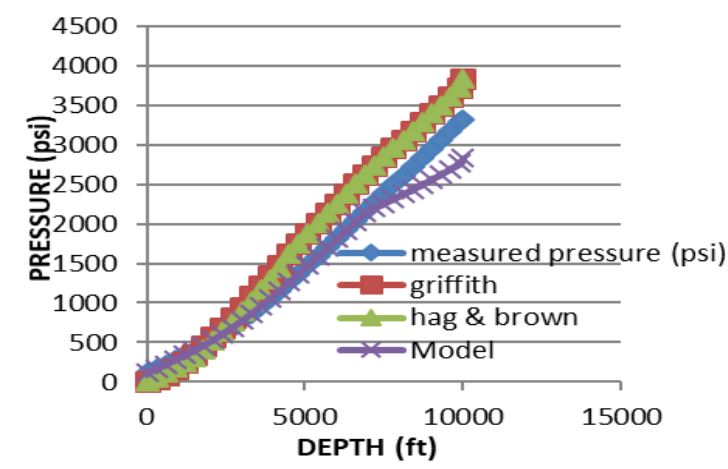

Fig. 3. Pressure along well.

The pressure curve for overall pressure drop gradient of 0.36 (psi/ft) and length of 10,000(ft) from the plot measured pressure was compared with predicted pressure from model and two correlations all the models followed the same path, but it was observed that was agreement of all the models at top of the well and Grifith \& Wallis and Hagedorn \& Brown over predicted at the depth of the well, the model was consistent to 70,000 (ft) depth before a deviation was noticed.

TABLE II: OVERALL PRESSURE GRADIENT

\begin{tabular}{ccccc}
\hline \hline $\begin{array}{c}\text { TVD } \\
(\mathbf{f t})\end{array}$ & $\begin{array}{c}\text { measured pressure } \\
(\mathbf{p s i})\end{array}$ & Griffith & $\begin{array}{c}\text { hag } \boldsymbol{\alpha} \\
\text { brown }\end{array}$ & Model \\
\hline $0-10,000$ & 0.320778 & 0.382649 & 0.382649 & 0.271514 \\
\hline \hline
\end{tabular}

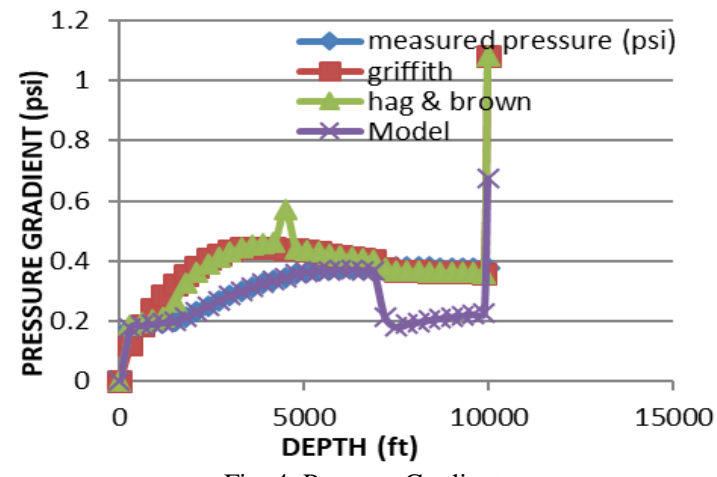

Fig. 4. Pressure Gradient.

Plot of measured pressure drop gradient and predicted models from $0-10,000(\mathrm{ft})$, total pressure drop decreases along the pipe, all the models predicted correctly at a point with the measured pressure drop, Griffith \& Wallis and Hageborn \& Brown over predicted after the point of agreement at top of the well, the measured and pressure model agreed and are consistent up 7000(ft) before a divergence was observed, all the model followed the same trend as the measured. The measured and the model pressure gradient at the bottom of the increased because the fluids entering the wellbore from reservoir can range from undersaturated oil to single phase gas. The in-situ gas velocity is insufficient to carry the produced liquid leading to liquid fallback in.

\section{CONCLUSION}

This study considered pressure drop gradient estimation using multiphase correlations and statistical model. The ability to predict flow behavior leads to accurate design of tubing and performance of oil and gas well. The efficient design of gas-lift pump, electric submersible pumps, separators, flow strings and other production equipment depends on the accurate prediction of the pressure drop along the flow pipe. Pressure is the energy of the well and it is crucial to understand how a change in fluid properties, flow conditions and pipe geometric properties affect this important parameter in the oil and gas industry. For easy and fast prediction, the statistical model has shown superiority to the empirical correlations because of their limitations.

A detail analysis of two phase flow in vertical well is carried out using field data, mathematical model, correlations and computer simulation to predict accurately the flow pattern, liquid holdup and pressure gradient for the optimization of well. From Fig. 1, it can be seen that the correlations and the measured pressure show a reasonable agreement, save at the depth of the well where the divergence is observed. In Fig. 2, different models were generated and quadratic model gave closer prediction to measured pressure drop and Table II showed the summary of pressure gradient in the well.

Statistical equation was generated to predict pressure drop gradient in multiphase flow in well. It is a better approach and an accurate model of predicting pressure gradient in multiphase flow in pipes.

Based on the analysis carried out in this work and from the conclusion drawn, Statistical model is highly recommended for multiphase pressure gradient prediction in 
vertical well.

Multiphase correlations can be used if statistical model is not available in predicting pressure drop gradient in multiphase flow in well.

\section{ACKNOWLEDGMENT}

The authors would like to appreciate the assistance of the staff of the software laboratory of the department of Mechanical Engineering, Rivers State University.

\section{REFERENCES}

[1] Saied, B. (2013). Prediction of Pressure Drop in vertical Air/water flow in the presence/Absence of Sodium DodecylSulfate as a surfactant. Thesis University of Dayton.

[2] Ideriah, F.J.K. (2017). $2^{\text {nd }}$ edition "Fundamentals of Fluid Mechanics" Vol. 1, Engineering Fluid Mechanics Series, T \& D Press Port Harcourt, Nigeria ( $1^{\text {st }}$ Edition 1986).

[3] Ogbeide P. O. and Igbinere S. A. (2015) Nodal Analysis Approach in Minimizing Pressure Losses to Improve Well Productivity Journal of Emerging Trends in Engineering and Applied Sciences (JETEAS) 6(5): 353- 359 C Scholarlink Research Institute Journals, 2015 (ISSN: 2141-7016) Petroleum Engineering Department, University of Benin, Benin City.

[4] Musaab, M. A. \& Mohammed A. A. (2014). A Comprehensive Study on the Current Pressure Drop Calculation in Multiphase Vertical Wells; Current Trends and Future Prospective. Journal of Applied Sciences, 14:3162-3171.

[5] Nwachuku, K.C. (2014). Detailed analysis of pressure drop in large diameter pipe. Msc Thesis Department of Petroleum Engineering, Africa University of Science and Technology, Nigeria.

[6] Adekomaya, O, Olafuruyi, O \&Blankson, E. (2011). An improved version of calcucating pressure transverse in multiphase flow in vertical pipe. Journal of petroleum \& coal (1),56-64.

[7] Omon, E., Dulu, A., \&Okotiem, A. (2015). Estimation of pressure Drop, Liquid Holodup and flow pattern in a Two phase vertical flow. International Journal of Engineering and Technology 5(4). Oregon State University.

[8] Masud, B. (1991). Most Accurate Two-Phase Pressure-Drop Correlation Identified. The Oil and gas journal

[9] Mukherjee, H. \& Brill, J. P. (1985). Pressure drop correlations for inclined two-phase flow. J. Energy Resour. Technol., 107: 549-554.

[10] Beggs, D.H. (2008). Production Optimization Using Nodal Analysis $2^{\text {nd }}$ edition, Oklahoma.

[11] Jain, A.K (1976) Accurate Explicit Equation for Friction Factor, J. Hydl. Div. ASCE, HY5

\section{NOMENCLATURE}

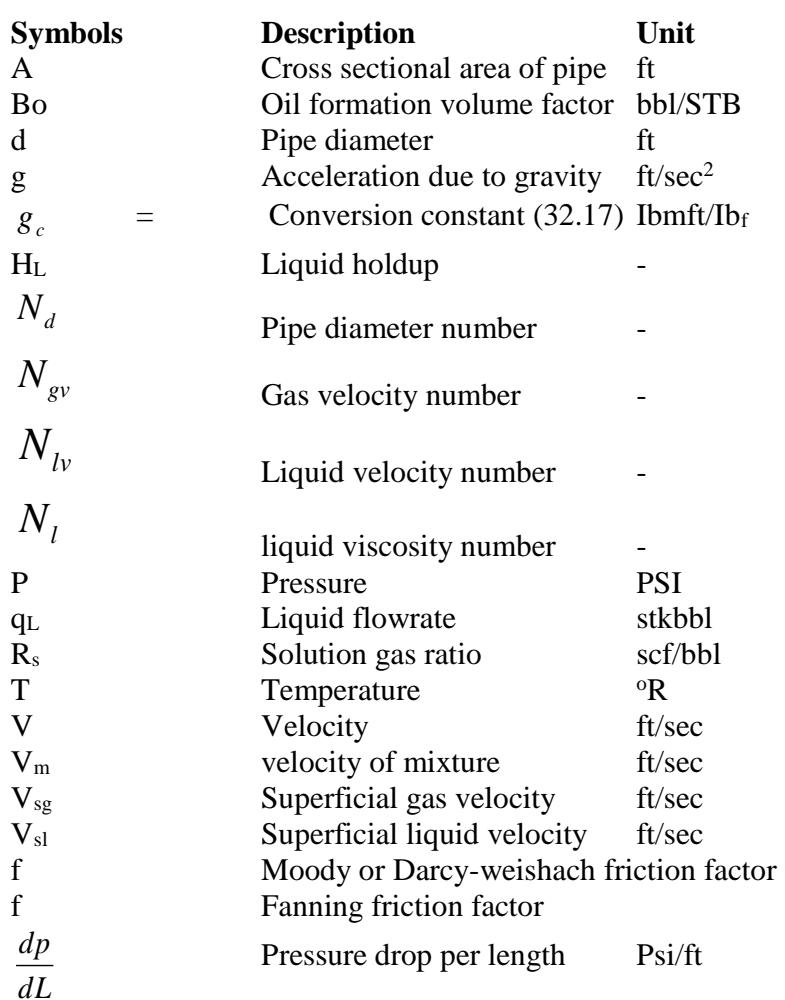

\section{ABBREVIATIONS}

GOR

GLR

Gas-Oil Ratio scf/stb

Gas-Liquid Ratio Scf/stb

Water-Oil Ratio

\section{SUBSCRIPTS}

Gas

Liquid

Mixture

Oil

Water 